\title{
The Synthesis and Characterization of Magnetic Chitosan-poly(N,N- diethylacrylamide) Semi-IPN Films
}

\author{
Burcu Aydoğdu, Ali Alipour, Gülten Gürdağ \\ Department of Chemical Engineering, Istanbul University \\ 34320 Avcilar, Istanbul, Turkey \\ burcu.aydogdu@ogr.iu.edu.tr; alialipour1985@ogr.iu.edu.tr; ggurdag@istanbul.edu.tr
}

\begin{abstract}
The aim of this study was to investigate the effect of $\mathrm{Fe}_{3} \mathrm{O}_{4}$ (magnetite) nanoparticles on the properties of magnetitecontaining chitosan-poly(N,N-diethylacrylamide) semi-interpenetrated network (CS-DE-M semi-IPN) films. For that purpose, nanosized $(\leq 50 \mathrm{~nm})$ magnetite-containing CS-DE-M semi-IPN films were prepared at four different initial N,N-diethylacrylamide concentrations. For the comparison purpose, CS-DE semi-IPN films without magnetite were also prepared under the same conditions with those of CS-DE-M films. The structural and thermal characterization of CS-DE-M and CS-DE films were performed by FTIR and DSC methods, respectively. The variation of swelling behavior of semi-IPN films with temperature was investigated in distilled water at four different temperatures between $10-40^{\circ} \mathrm{C}$ with step of $10^{\circ} \mathrm{C}$. The equilibrium swelling values of CS-DE films were found to be three times higher than those of CS-DE-M films at all investigated temperatures. The swelling values of both polymer films decreased with the increase in DE concentration in CS solution. In addition, the lowest swelling was observed at $10^{\circ} \mathrm{C}$, and it increased with the increase in temperature in contrary to the expected results from a thermo-responsive polymer such as poly(N,N-diethylacrylamide). Normally, the swelling of a thermoresponsive polymer decreases with temperature, in the case of CS-DE and CS-DE-M films, however, the maximum poly(N,N-diethylacrylamide) content is between $27 \%$ and $57 \%$, and it is too low to display a response to temperature as shrinking.
\end{abstract}

Keywords: Chitosan, poly(N,N-diethylacrylamide), magnetite, swelling, DSC, FTIR

\section{Introduction}

Chitosan (CS) is a good-film forming, non-toxic, biodegradable, and biocompatible polymer, and it is a linear chained copolymer of D-glucosamine and N-acetyl-D-glucosamine. CS dissolves in acidic solutions by the protonation of its amine groups, and for that reason, it can be classified as a cationic polymer. Since the protonation of amine groups of $\mathrm{CS}$ varies with the $\mathrm{pH}$ of medium, it is a $\mathrm{pH}$-responsive polymer which responds to the changes in $\mathrm{pH}$ of external medium reversibly. Due to several valuable properties such as antibacterial, antifungal, biodegradability as well as film-, fiber- and hydrogel-forming capability, and biocompatibility, it is used in diverse biomedical applications [1].

Poly(N,N-diethylacrylamide) (PDE) hydrogel is a typical thermo-sensitive polymeric network, which exhibits a lower critical solution temperature (LCST) at about $31^{\circ} \mathrm{C}$ [2]. The swelling and drug-delivery properties of poly( $\mathrm{N}$-isopropyl acrylamide) (PNIPAM) and poly(N,N-diethyl acrylamide) (PDE) gels were compared, and found that PDE-based gels have a broader phase transition temperature interval than PNIPAM-based gels [2]. Zhang et al. [3] compared the properties of poly(2-dimethylaminoethyl methacrylate-co-poly(N,N-diethyl acrylamide) (P(DMAEMA-co-DE)) semi-IPN and PDE gels. They have found that $\mathrm{P}(\mathrm{DMAEMA}-\mathrm{co}-\mathrm{DE})$ gels responded faster to the changes in $\mathrm{pH}$ and temperature than PDE ones. Ngadaonye et al. [4] reported that thermo-responsive CS-PDE films displayed a negative temperature-dependent swelling and an efficient delivery of antibiotics for wound dressing applications.

Nano-sized magnetite (M), which is an iron (II,III) oxide $\left(\mathrm{Fe}_{3} \mathrm{O}_{4}\right)$, has been widely used in recent biomedical applications. Magnetite-containing CS gels are finding new biomedical applications such as cancer treatment and biosensors. It has been known that the structural modification of CS gels by $\mathrm{Fe}_{3} \mathrm{O}_{4}$-introduction enhances the activity and biodegradability of gels. Cesano et al. [5] reported that CS-M composite films can be used in environmental applications such as the removal of pollutants from solid or liquid media due to their magnetic property. Ye et al. [6] showed that magnetite/graphene/chitosan (M/GO/CS) composites can be used as magnetic adsorbent for protein and it had a promising potential in magnetic separation process. Finotelli et al. [7] reported that magnetite-containing alginate/chitosan beads 
delivered less insulin than alginate beads. The preparation of magnetite-chitosan (CS-M) nanoparticles by the coating of $\mathrm{CS}$ on the magnetic $\mathrm{Fe}_{3} \mathrm{O}_{4}$ nanoparticles were also performed $[8,9]$.

As can be seen in the works summarized above, there are composite polymer structures prepared from either CS and $\mathrm{DE}$ or CS and M, but there is no published work containing all the three constituent of CS-DE-M film together. In the light of this finding, CS-DE-M semi-IPN polymer films were prepared by the polymerization of DE at different initial monomer concentrations in the presence of crosslinker in the solutions of CS and their swelling and thermal properties were compared with those of CS-DE semi-IPN films in order to determine the presence of magnetite in the polymer structure.

\section{Experimental}

\subsection{Materials}

CS (low viscosity) and the crosslinker N,N'-methylenebiscrylamide (NMBA) were purchased from Sigma. N,Ndiethylacrylamide (DE) monomer was obtained from Polysciences, Inc (Germany). Magnetite $\left(\mathrm{Fe}_{3} \mathrm{O}_{4}\right)(\mathrm{M})(\leq 50 \mathrm{~nm})$ was of Aldrich product. The initiator ammonium persulfate (APS) and the accelerator N,N,N',N'-tetramethylethylenediamine (TEMED) were purchased from Riedel-de Haen (Seelze, Germany) and Serva Electrophoresis GmbH (Heidelberg, Germany), respectively. For the preparation of semi-IPN films and swelling measurements, distilled water was used.

\subsection{Synthesis of magnetic CS-DE semi-IPN films}

Magnetite-containing CS-DE (CS-DE-M) semi-IPN films were prepared by a solution casting and solvent evaporation technique. At first, CS $(0.5 \mathrm{~g})$ was dissolved in $25 \mathrm{~mL}, 2 \%(\mathrm{v} / \mathrm{v})$ aqueous acetic acid at room temperature by stirring with a magnetic stirrer. Then, $0.01 \mathrm{~g} \mathrm{Fe}_{3} \mathrm{O}_{4}(\mathrm{M})$ was added to that CS solution and dispersed by ultrasonic stirrer by keeping the temperature below $50^{\circ} \mathrm{C}$ for 1 hour. Afterwards, cross-linker NMBA, the monomer DE, the initiator APS, and the accelerator TEMED were added to the M-containing CS solution, respectively, and the mixture was re-dispersed by ultrasonic stirrer in order to obtain a completely homogeneous mixture for $3 \mathrm{~min}$. The viscous reaction mixture was cast into a Teflon petri dish with inner diameter of $10 \mathrm{~cm}$, and left at room temperature for $5 \mathrm{~min}$. Then, the Teflon petri dish was covered with parafilm, placed in the freezer unit of a refrigerator, and held at $-18^{\circ} \mathrm{C}$ for 48 hours. After that time, petri dish was taken out of the refrigerator, and kept at room temperature in order to obtain CS-DE-M films by the polymerization of $\mathrm{DE}$, and the evaporation of water.

For the comparison, CS-DE semi-IPN films were also prepared under the same conditions with those of CS-DE-M films. Polymer films were synthesized at four different DE initial monomer concentrations in CS solution, and they were coded as CS-xDE-M, where " $x$ " denotes the initial DE concentration in CS solution. The polymer film codes and their feed composition were given in Table 1 .

Table 1: The codes and compositions of CS-DE-M semi-IPN films.

\begin{tabular}{|c|c|c|c|c|c|c|}
\hline Film code & $\begin{array}{l}\text { CS } \\
(\mathrm{g})\end{array}$ & $\begin{array}{c}{[\mathrm{DE}]^{\mathrm{a}}} \\
(\mathrm{mol} / \mathrm{L})\end{array}$ & $\begin{array}{c}\mathrm{Fe}_{3} \mathrm{O}_{4} \\
(\mathrm{~g})\end{array}$ & $\begin{array}{l}\mathrm{NMBA}^{\mathrm{b}} \\
(\mathrm{mol} \%)\end{array}$ & $\begin{array}{c}\text { APS }^{\mathbf{c}} \\
(\mathrm{mol} \%)\end{array}$ & $\begin{array}{c}\text { TEMED }^{\mathrm{d}} \\
\text { (g) }\end{array}$ \\
\hline CS-0.06DE-M & 0.50 & 0.06 & 0.01 & 5 & 1 & WAPS \\
\hline CS-0.12DE-M & 0.50 & 0.12 & 0.01 & 5 & 1 & W $_{\text {APS }}$ \\
\hline CS-0.18DE-M & 0.50 & 0.18 & 0.01 & 5 & 1 & WAPS \\
\hline CS-0.21DE-M & 0.50 & 0.21 & 0.01 & 5 & 1 & W $_{\text {APS }}$ \\
\hline
\end{tabular}

a: $[D E]$ is the initial concentration of DE monomer in CS solution $(25 \mathrm{~mL})$ in mol/L. b: NMBA was used in 5 mol \% of DE monomer; c: APS was used in $1 \mathrm{~mol} \%$ of DE monomer; d: TEMED was used in equal amount of APS in weight.

\subsection{Characterization of CS-DE-M and CS-DE semi-IPN gel films}

Both CS-DE-M and CS-DE films were characterized by the KBr pellet method between 400 and $4000 \mathrm{~cm}^{-1}$ using Spectrum One, Perkin-Elmer (USA) FTIR equipment.

Differential scanning calorimetry (DSC) analysis of vacuum-dried films were recorded by using DSC 131, Setaram equipment. DSC curves given in the figures were obtained by the procedure as follows: first, the dry film sample (about 15 
$\mathrm{mg}$ ) was heated to $150^{\circ} \mathrm{C}$ at $10^{\circ} \mathrm{C} / \mathrm{min}$ under nitrogen flow rate of $40 \mathrm{~mL} / \mathrm{min}$, and then it was cooled to $30^{\circ} \mathrm{C}$. Second, the sample was reheated from $30^{\circ} \mathrm{C}$ to $350^{\circ} \mathrm{C}$ at the same heating rate under nitrogen gas atmosphere with the same flow rate. This procedure ensured that all the samples had the same thermal history.

The equilibrium swelling values (ESVs) of CS-based magnetic semi-IPN films were determined by gravimetric method in distilled water at four different temperatures between $10-40^{\circ} \mathrm{C}$ with step of $10^{\circ} \mathrm{C}$. A given amount of dry film (Wd) was immersed in distilled water at determined temperature for $24 \mathrm{~h}$ in order to attain in swelling equilibrium. Swollen gel films were taken out of from water, blotted with a piece of filter paper to remove excess water, and weighed (Ws). ESVs of the films were calculated using the following equation:

$$
\operatorname{ESV}\left(\mathrm{gH}_{2} \mathrm{O} / \mathrm{g}_{\text {polymer }}\right)=\left(W_{s}-W_{d}\right) / W_{d}
$$

where $W_{s}$ and $W_{d}$ are the weights of swollen and dry films, respectively.

\section{Results}

FTIR spectra of CS, CS-DE, and CS-DE-M films were given in the Figures 1-2. The increase in the intensity of the band attributed to the stretching vibration of $\mathrm{C}=\mathrm{O}$ bonds in PDE confirmed the presence of DE network in both CS-DE $\left(1627 \mathrm{~cm}^{-1}\right)$ and CS-DE-M $\left(1642 \mathrm{~cm}^{-1}\right)$ films. While the band due to $\mathrm{Fe}-\mathrm{O}$ bond in $\mathrm{Fe}_{3} \mathrm{O}_{4}$ is seen at $587 \mathrm{~cm}^{-1}$ [1], it is not seen in the spectra of CS-DE-M films due to their $\mathrm{Fe}_{3} \mathrm{O}_{4}$ content below the detection limit (Figure 2).

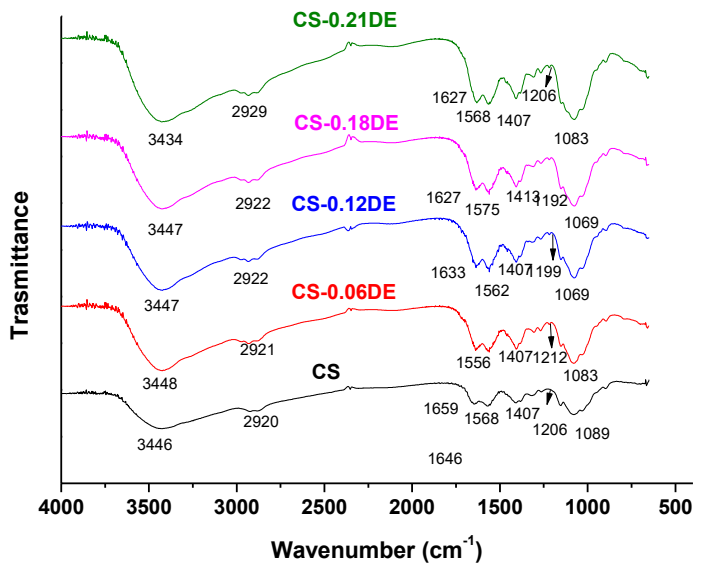

Fig. 1: FTIR spectra of CS and CS-DE semi-IPN films.

ESVs of CS-DE-M and CS-DE semi-IPN films in distilled water were given in Figures 3 and 4, respectively. The presence of magnetite in CS-DE films decreased the swelling values of films. ESVs of CS-DE films were found to be three times higher than those of CS-DE-M films at all investigated temperatures. The swelling values of both polymer films decreased with the increase in PDE content of polymer due to the increase in the hydrophobicity of the semi-IPN structure. In addition, the lowest swelling was observed at $10^{\circ} \mathrm{C}$, and it increased with the increase in temperature in contrary to the expected results from a thermo-responsive polymer such as poly(N,N-diethylacrylamide). Normally, the swelling of a thermo-responsive polymer decreases with temperature, in the case of CS-DE and CS-DE-M films, however, the maximum poly(N,N-diethylacrylamide) content is between $27 \%$ and $57 \%$, and it is too low to display a response to temperature as shrinking. It is also known that the increase in hydrophilic polymer content in a thermo-responsive NIPAM copolymer leads to the decrease and finally disappear in the thermo-sensitivity of the polymer. Since CS is more hydrophilic than PDE network, the amount of PDE in CS-DE or CS-DE-M films was not enough to display thermo-sensitivity. At higher DE initial concentrations than $0.21 \mathrm{M}$, a semi-IPN structure was not obtained since the polymerization and crosslinking of DE in CS solution was not completed. 


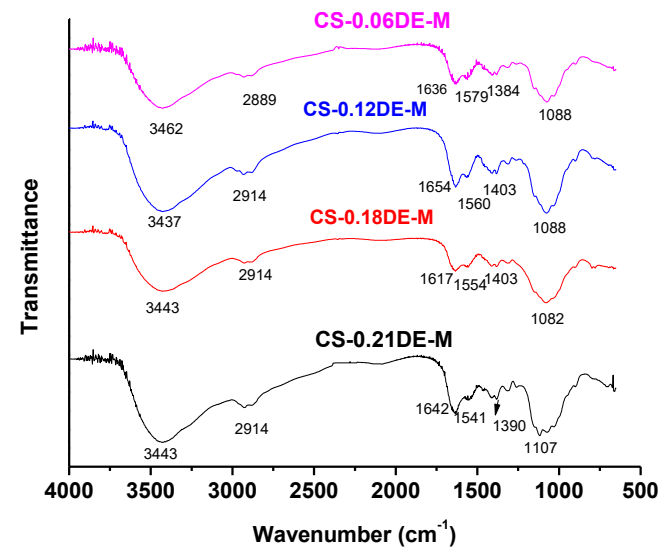

Fig. 2: FTIR spectra of CS-DE-M semi-IPN films.

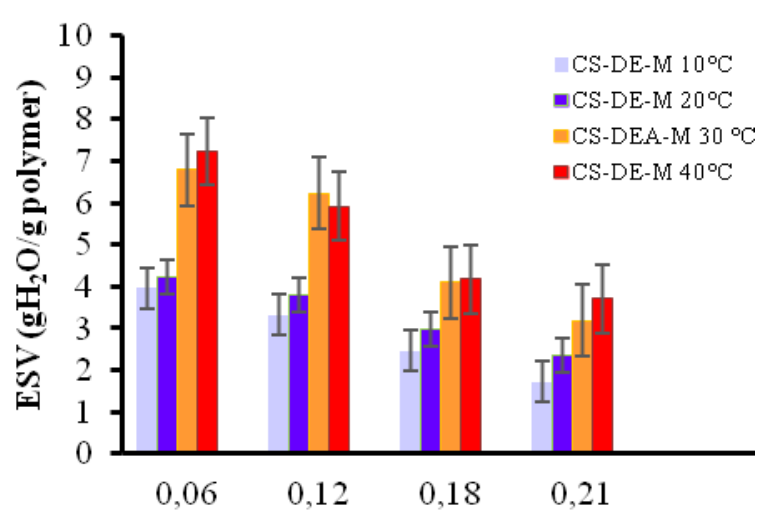

$\lceil\mathrm{DE}\rceil(\mathbf{m o l} / \mathbf{L})$

Fig. 3: The variation of equilibrium swelling values of CS-DE-M semi-IPN films in distilled water with temperature.

It is known that thermal decomposition of $\mathrm{CS}$ begins at the temperatures above $250^{\circ} \mathrm{C}$ [10], and the main decomposition of CS in CS-M films occurs at $285^{\circ} \mathrm{C}$, and it does not change with the increase in magnetite content from 1 to $5 \%$ in CS-M films [1]. In one of our previous works [10], we prepared semi-IPN films from CS and N,Ndimethylacrylamide (DA) without $\mathrm{Fe}_{3} \mathrm{O}_{4}$ at the same initial DA monomer concentrations with those of CS-DE-M or CS-DE films, namely at $0.06,0.12,0.18$ and $0.21 \mathrm{M}$ initial DA concentrations, and performed their thermal characterization by DSC [10]. DSC analysis of these films showed that the presence and the further increase in cross-linked PDA content in CS-DA semi-IPN films decreased the maximum decomposition temperature from $261^{\circ} \mathrm{C}$ to $240^{\circ} \mathrm{C}$, most probably due to breaking of intra- and intermolecular hydrogen bonds between CS chains. In addition, it was also observed that pristine CS film is thermally more stable than CS-DA semi-IPN films [10]. The peak decomposition temperatures corresponding to the main decomposition of CS backbone in CS-DE and CS-DE-M films were given in Table 2. The presence of PDE in the polymer structure and its further increase in CS-DE films decreased the main decomposition temperature of CS from $295^{\circ} \mathrm{C}$ to $265^{\circ} \mathrm{C}$, namely decreased the thermal stability of (Table 2). This finding is suitable with those obtained for CSDA semi-IPN films. 


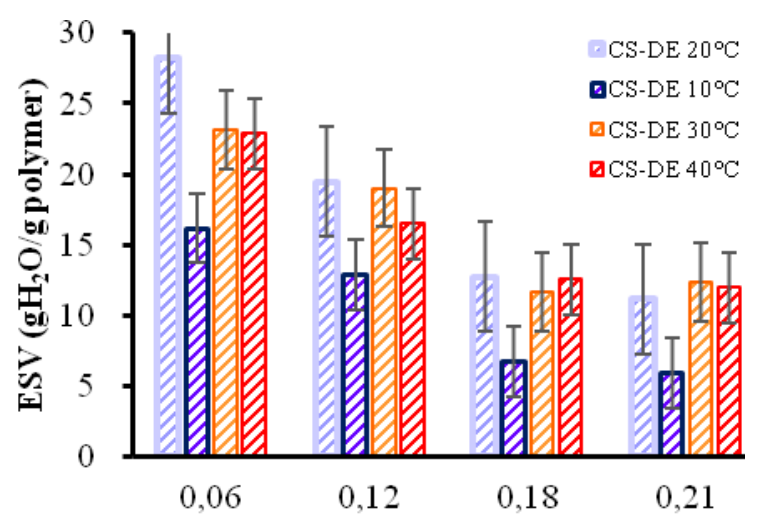

$\lceil\mathrm{DE}\rceil(\mathrm{mol} / \mathrm{L})$

Fig. 4: The variation of equilibrium swelling values of CS-DE semi-IPN films in distilled water with temperature.

Table 2: The peak decomposition temperatures of CS-DE-M and CS-DE semi-IPN films.

\begin{tabular}{|c|c|c|}
\hline \multirow{2}{*}{$\begin{array}{c}{[\mathbf{D E}]} \\
\left(\mathbf{m o l . L}^{-\mathbf{1}}\right)\end{array}$} & \multicolumn{2}{|c|}{$\begin{array}{c}\text { Peak Decomposition Temperature } \\
\left({ }^{\mathbf{0}} \mathbf{C}\right)\end{array}$} \\
\cline { 2 - 3 } & $\mathbf{C S}-\mathrm{DE}$ & $\mathbf{C S}-\mathrm{DE}-\mathbf{M}$ \\
\hline 0.06 & 295 & 256 \\
\hline 0.12 & 275 & 263 \\
\hline 0.18 & 269 & 270 \\
\hline 0.21 & 265 & 269 \\
\hline
\end{tabular}

In the case of magnetite-containing CS-DE-M films, it was no observed a great change between the peak decomposition temperatures with the increase in PDE content of the semi-IPN structure. The slightly lower thermal stability of the CS-DE-M films prepared at low $(0.06$ and $0.12 \mathrm{M}) \mathrm{DE}$ initial concentrations can be attributed to the presence of magnetite in the polymer film. The small change in the peak decomposition temperatures of CS-DE-M films can also be attributed to the stabilization effect of magnetite which is present in the films. It was also determined that the peak decomposition temperatures did not change with the increase between 1 to $5 \mathrm{wt} \%$ in $\mathrm{M}$ content of the CS-M films, and they were remained constant at $285^{\circ} \mathrm{C}$ [10]. This finding is suitable with that of our present finding, namely the thermal stabilization effect of magnetite in CS-DE-M films.

\section{Conclusion}

CS-DE-M and CS-DE films did not display a thermo-responsive property due to low content of PDE in the polymer structure. The presence of DE network in CS-DE and CS-DE-M semi-IPN films decreased the swelling values. The presence of magnetite in CS-DE films decreased the swelling values of films. The swelling of CS-DE-M films was three times lower than those of CS-DE films. While the increase in PDE content of CS-DE films decreased the thermal stability of the films, in the case of magnetite-containing films, it enhanced the thermal stability of the films, and led to the increase in the peak decomposition temperatures from $256^{\circ} \mathrm{C}$ to $268^{\circ} \mathrm{C}$ for CS-DE-M films most probably due to thermal stabilization effect of magnetite. This finding is suitable with the finding determined in a previous MSc Thesis [1].

\section{Acknowledgements}

This paper was derived from MSc Thesis of Burcu Aydoğdu prepared in Chemical Engineering Department of Istanbul University. This work was supported for presentation in ICNNFC'16 by the Research Fund of Istanbul University with project number \#BYP 49439. 


\section{References}

[1] H. Filiz, "The Preparation and characterization of chitosan-metal oxide composite films," M.Sc. thesis, Dept. Chem. Eng., Istanbul Univ., Istanbul, Turkey.

[2] M. Panayiotou and R. Freitag, "Synthesis and characterisation of stimuli-responsive poly(N,N'- diethylacrylamide) hydrogels," Polymer, vol. 46, pp. 615-621, 2005.

[3] N. Zhang, M. Liu, Y. Shen, J. Chen, L. Dai, and C. Gao, "Preparation, properties, and drug release of thermo- and pH sensitive poly ((2dimethylamino) ethyl methacrylate)/ poly(N,N-diethylacrylamide) semi-IPN hydrogels," J. Mater. Sci., vol. 46, pp. 1523-1534, 2011.

[4] J. I. Ngadaonye, L. M. Geever, K. E. McEvoy, J. Killion, D. B. Brady, and C. L. Higginbotham, "Evaluation of novel antibiotic-eluting thermoresponsive chitosan-pdeaam based wound dressings," Int. J. Polym. Mater. Polym. Biomater., vol. 63, pp. 873-883, 2014.

[5] F. Cesano, G. Fenoglio, L. Carlos, and R. Nistico, "One-step synthesis of magnetic chitosan polymer composite films," Appl. Surf. Sci., vol. 345, pp. 175-181, 2015.

[6] N. Ye, Y. Xie, P. Shi, T. Gao, and J. Ma, "Synthesis of magnetite/graphene oxide/chitosan composite and its application for protein adsorption," Mater. Sci. Eng. C, vol. 45, pp. 8-14, 2014.

[7] P. V. Finotelli, D. Da Silva, M. Sola-Penna, A. M. Rossi, M. Farina, L. R. Andrade, A. Y. Takeuchi, and M. H. Rocha-Leao, "Microcapsules of alginate/chitosan containing magnetic nanoparticles for controlled release of insulin," Colloids Surf. B:Biointerfaces, vol. 81, pp. 206-211, 2010.

[8] G.-y. Li, Y.-r. Jiang, K.-l. Huang, P. Ding, and J. Chen, "Preparation and properties of magnetic $\mathrm{Fe}_{3} \mathrm{O}_{4}$-chitosan nanoparticles," J. Alloys Compounds, vol. 466, pp. 451-456, 2008.

[9] A. A. Kadam, and D. S. Lee, "Glutaraldehyde cross-linked magnetic chitosan nanocomposites: Reduction precipitation synthesis, characterisation, and application for removal of hazardous textile dyes,"Bioresource Techno., vol. 193, pp. 563-567, 2015.

[10] S. Sarmad, G. Yenici, K. Gürkan, G. Keçeli, and G. Gürdağ, "Electric field responsive chitosan-poly(N,N-dimethyl acrylamide) semi-IPN gel films and their dielectric, thermal and swelling characterization," Smart. Mater. Struct., vol. 22, no. 5, 055010, pp. 1-15, 2013. 